\title{
LOS JUEGOS OLÍMPICOS DE LA JUVENTUD Y LAS REDES SOCIALES (2010-2014): EVOLUCIÓN Y RETOS EN LAS NUEVAS FORMAS DE COMUNICACIÓN PARA LA TRANSMISIÓN DE VALORES OLÍMPICOS
}

\author{
José M. Pardo Gila \\ Universidad Autónoma de Barcelona \\ gilajose@gmail.com \\ María Teresa Calle Molina \\ Universidad Autónoma de Madrid \\ mariat.calle@uam.es
}

Fecha de recepción: Marzo 2016

Fecha de aceptación: Octubre 2016

http://dx.doi.org/10.15366/citius2016.9.2.002

Resumen:

El nacimiento de los Juegos Olímpicos de la Juventud coincide en el tiempo con la irrupción de las redes sociales proporcionando nuevos canales para divulgar las ideas pedagógicas de Pierre de Coubertin, el fundador de los Juegos Olímpicos modernos. Nankín 2014 supuso la consolidación del Movimiento Olímpico en las redes explorando nuevos mercados y estrategias que alcanzaron los 500 millones de usuarios en todo el mundo. La concepción de los medios sociales como herramientas enraizadas al ámbito local en un contexto global permitirá a las instituciones deportivas globales el diseño de estrategias de comunicación más directas, específicas e individualizadas de acuerdo a las particularidades de sus públicos.

Palabras clave: Olimpismo; Juegos Olímpicos Juventud; comunicación digital; redes sociales.

Title: THE YOUTH OLYMPIC GAMES AND SOCIAL NETWORKS (2010-2014): EVOLUTION AND CHALLENGES OF NEW METHODS OF COMMUNICATION FOR THE TRANSMISSION OF OLYMPIC VALUES

\begin{abstract}
:
The birth of the Youth Olympic Games coincided with the emergence of social networks, providing new channels for disseminating the pedagogical ideas of Pierre de Coubertin - the founder of the modern Olympic Games. Nanjing 2014 served as the consolidation of the Olympic Movement in social networks, exploring new markets and strategies that reached 500 million users around the world. Understanding social media as a tool rooted at local level in a global context will allow global sporting institutions to design communication strategies that are more direct, specific and individualized, according to the particularities of their audiences.
\end{abstract}

Key words: Olympism; Youth Olympic Games; digital communication; social networks

\section{Introducción}

El deporte representa un pilar fundamental en la vida recreativa de millones de personas por su capacidad para involucrar a grandes públicos, además de tener influencia en el desarrollo de la tecnología de los medios tradicionales y un rol destacado con la llegada de internet (Billings, 
2010). Los medios sociales han supuesto un nuevo escenario en el mundo del deporte y su relación con los jóvenes por la capacidad estratégica para conectar audiencias y para que éstas difundan los valores e ideales olímpicos (Fernández Peña, Ramajo y Arauz, 2014). Si la televisión transformó los Juegos Olímpicos (JJOO) y los condujo a una audiencia global (Moragas, Rivenburgh y Larson, 1995), los medios sociales han extendido este alcance construyendo nuevos lazos especialmente con los jóvenes (Fernández Peña y Ramajo, 2014).

Los Juegos Olímpicos de la Juventud nacen en este contexto como un proyecto propuesto por el expresidente del Comité Olímpico Internacional (COI), Jacques Rogge, aprobado el 5 de julio de 2007 durante la 119 a sesión del COI celebrada en Guatemala (COI, 2007; Judge, Petersen y Lydum, 2009). La creación de este evento deportivo tiene como objetivos principales motivar a los jóvenes en la práctica del deporte, promover los valores olímpicos y difundir el mensaje del Movimiento Olímpico entre los jóvenes de todo el mundo (COI, 2014a; Wong, 2011). La educación y la cultura son los componentes clave para comprender la concepción de los Juegos Olímpicos de la Juventud (COI, 2011; Jackson, 2014). A partir del Programa de Cultura y Educación, impulsado por el COI y celebrado durante cada edición de los Juegos Olímpicos de la Juventud (JOJ), los deportistas participan en actividades que promueven los valores olímpicos de la excelencia, la amistad y el respeto, y aprenden sobre los beneficios del deporte, el estilo de vida saludable, la responsabilidad social y las nuevas formas de comunicación (COI, 2010a). El énfasis en este tipo de actividades enfocadas en la educación y la cultura es lo que diferencia a este mega evento deportivo (COI, 2011; COI, 2014a). Es esa idea pedagógica del olimpismo de Pierre de Coubertin, con los jóvenes siempre tan presentes en sus escritos, el origen de la creación de los Juegos Olímpicos de la Juventud puesta en práctica por Jacques Rogge.

Estudiar el olimpismo en relación a los jóvenes en las redes sociales es incidir en dos aspectos que se complementan de forma fértil y dinámica: se analizan unos Juegos Olímpicos centrados en los más jóvenes, pero también unos nuevos medios que subsisten gracias a la participación de los públicos y sus producciones. Los jóvenes, que utilizan estos medios masivamente y de forma activa —el 92\% de los adolescentes de entre 13 y 17 años residentes en Estados Unidos utiliza internet a diario y el $71 \%$ está online en más de una red social (Lenhart, 2015 , p. 2-3) - , han entendido como casi nadie la naturaleza de las redes sociales.

Este trabajo, por tanto, analiza dos caminos paralelos: el del surgimiento de los Juegos Olímpicos de la Juventud y el de la introducción de las redes sociales como herramienta de comunicación con los más jóvenes, y se adentra en las lógicas que alimentan la cultura de comunicación en este evento. Al mismo tiempo, puesto que en el ADN de estos nuevos medios está inscrito su vocación de participación, también analizamos la presencia de los públicos siguiendo la evolución histórica en las ediciones celebradas de los Juegos de la Juventud (Singapur 2010 y Nankín 2014, en sus ediciones en verano, e Innsbruck 2012, en su primera edición de invierno) y planteamos algunas de las cuestiones que debe considerar el Movimiento Olímpico de cara a las próximas ediciones en Lillehammer 2016, Buenos Aires 2018 y Lausana 2020.

Los objetivos de este artículo son:

1. Analizar cronológicamente la evolución histórica del Movimiento Olímpico en las redes sociales.

2. Investigar la evolución de la estrategia de la presencia en las redes sociales durante las tres ediciones celebradas de los Juegos Olímpicos de la Juventud (Singapur 2010, Innsbruck 2012 y Nankín 2014). 


\section{Metodología}

Previa categorización de las diversas fuentes utilizadas, que ha permitido establecer y priorizar los datos de fuentes primarias y secundarias, este artículo se fundamenta en la revisión y el análisis de los informes, documentos y contenidos oficiales de los Juegos Olímpicos de la Juventud publicados por el Comité Olímpico Internacional, así como en los libros y artículos académicos publicados sobre el objeto de estudio.

La metodología aplicada se apoya en la técnica del análisis de documentos que, como afirma Ruiz Olabuénaga (2012, p. 193), "se basa en la lectura como instrumento de recogida de información; lectura que ha de realizarse de modo científico, es decir, de manera sistemática, objetiva, replicable, válida".

\section{El olimpismo entre los jóvenes y la era de la comunicación digital}

La llegada de internet ha supuesto grandes cambios no sólo en la forma, sino en la naturaleza de la comunicación (Castells, 2001). A través de internet, la comunicación ha derribado el monopolio que concentraban los medios de comunicación, las instituciones y las grandes empresas (Levine, Locke, Searls y Weinberger, 2008). Desde mediados de los años noventa del siglo pasado los usuarios de la comunicación han ido adquiriendo progresivamente un papel protagonista. El advenimiento de los medios sociales ha sido el colofón a este creciente protagonismo de los públicos. Organizaciones internacionales, marcas globales, entidades gubernamentales y personajes mediáticos comparten ahora la primera línea informativa con los usuarios (Jenkins, 2008). El deporte y su entorno no ha sido ajeno a este nuevo panorama en el que internet se erige como foco de la realidad. Organizaciones deportivas, medios de comunicación, organizaciones comerciales y fans/consumidores conforman un ecosistema complejo que se retroalimenta (Moragas, Kennett y García Sedó, 2004). En este escenario, el Comité Olímpico Internacional, pionero en la introducción de avances y nuevas tecnologías como la televisión (Moragas et al., 1995), ha sido precursor en la explotación de las redes sociales dentro del ámbito del deporte (Fernández Peña, 2011).

"La difusión directa de mensajes más cercanos y sinceros sin intermediarios ha supuesto un acercamiento entre las instituciones deportivas, atletas de élite y los fans, y han convertido a éstos últimos en divulgadores de los valores olímpicos" (Fernández Peña et al., 2014, p. 166).

\subsection{Génesis y evolución de los Juegos Olímpicos de la Juventud}

El instaurador y precursor de los Juegos Olímpicos modernos, Pierre de Fredi Barón de Coubertin "era sobre todo un pedagogo, un educador, un filántropo, un humanista, que siempre había concebido al deporte y a la gran fiesta olímpica como generadores de paz y cultura" (Durántez Corral, s.f.: 6). Por ello, Pierre de Coubertin, en su faceta pedagógica afirmaba que "enseñar no era educar. Entre la enseñanza que da conocimientos, provee el espíritu y hace sabios, y la educación que desarrolla las facultades, eleva el alma y hace hombres, hay una diferencia profunda" (Coubertin, 2012, p. 134). Sin duda, para él los Juegos Olímpicos en su génesis eran una herramienta pedagógica fundamental, en especial, para las nuevas generaciones.

Siguiendo la línea ideológica de Pierre de Coubertín, los Juegos Olímpicos de la Juventud, fueron propuestos por el COI y aprobados en julio del 2007, apoyándose en los Principios Fundamentales de la Carta Olímpica Internacional (COI, 2014a). A través de estos valores, el Movimiento Olímpico trata de "reforzar, promover y apoyar a la juventud de todo el mundo, independientemente de su raza, religión y género y no solo a los mejores atletas, sino a 
aquellos que compiten de acuerdo a los principios fundamentales del olimpismo" (Rogge, 2001, en Makris y Georgiadis, 2013, p. 92).

Dos antecedentes a los JOJ fueron el Festival Olímpico de la Juventud Europea, que en 1991 tuvo su acto inaugural en Bélgica y en 1933 su homónimo invernal en Italia y el Festival Olímpico de la Juventud en Australia (Wong, 2011) propuestos tras los JJOO de Sydney 2000; siendo ambos festivales bienales. Del mismo modo, los JOJ se celebran cada dos años alternando las ediciones de verano e invierno, con una duración de 12 y 10 días respectivamente dirigido por y para jóvenes (COI, 2007) de entre 15 y 18 años (COI, 2014a).

La naturaleza de estos Juegos tiene como objetivo impulsar nuevas ideas, como incorporar nuevos formatos de competición o desarrollar un programa deportivo adaptándose al país de acogida de los Juegos, pudiendo variar en sus distintas ediciones (COI, 2014a). Hasta 28 disciplinas deportivas conforman el programa de verano, mientras que el de invierno consta de siete modalidades deportivas, incluyendo en el programa otras disciplinas y formatos no institucionales (COI, 2009), sirviendo, así, como plataforma de exhibición y difusión de otros deportes que no están recogidos en el programa oficial (COI, 2014a). Además, estos Juegos incluyen un programa de educación y cultura, también conocido como "Learn \& Share activities" (COI, 2014a, p. 3), que trata cinco ejes principales: olimpismo, desarrollo de habilidades, bienestar y vida sana, responsabilidad social y expresión (Ibídem). Este programa es una de las partes fundamentales de este mega evento como demuestra la edición de Nankín 2014, donde se realizaron 1.498 actividades incluidas en este programa durante su celebración (2014d).

Además, otros participantes que no son atletas profesionales tienen cabida en este programa, como "The Young Reporters" (COI, 2013, p. 1), jóvenes periodistas que han comenzado o han terminado la formación universitaria de periodismo y tienen entre 18 y 24 años. Son seleccionados por su respectivo Comité Olímpico Nacional, pero finalmente cada continente sólo puede seleccionar a cuatro de ellos con una representación de cada género del $50 \%$. Estos jóvenes reciben formación teórica y práctica en este evento con la ayuda de periodistas profesionales (COI, 2013). Por otro lado, "The Young Ambassadors" (COI, 2014e, p. 2) son 30 jóvenes de 18 a 28 años que también han sido seleccionados por su Comité Olímpico Nacional. Estos programas les ofrecen actividades antes y durante los Juegos (COI, 2010a), en los que trabajan para conocer los deportes, la gestión del tiempo, la nutrición, el medio ambiente, la responsabilidad y la ética de los medios, cuyas misiones principales son promover y difundir los JOJ en sus países y a través de las redes sociales defendiendo un estilo de vida saludable (COI, 2014a). Por último, "The Athlete Role Models" (COI, 2014e, p. 2) son aquellos campeones olímpicos elegidos por la federación internacional de su disciplina deportiva que participan de manera activa en el asesoramiento a los jóvenes en sus experiencias como atletas y en la vida que llevan como deportistas de élite (COI, 2014e).

El futuro de este conjunto de programas y soportes educativos y culturales están acogidos en el documento "Olympic Agenda 2020" (COI, 2014c). Este documento fue acordado por unanimidad en la $127^{\mathrm{a}}$ sesión del Comité Olímpico Internacional celebrado en Mónaco en diciembre del 2014 (Ibídem). Catorce grupos de trabajo sintetizaron unas 1.200 ideas que provenían de los debates generados en el Movimiento Olímpico y la sociedad en general elaborando y publicando 40 recomendaciones de mejora (Ibídem). La importancia que el COI otorga a los Juegos Olímpicos de la Juventud se recoge en algunas de estas recomendaciones tales como la inclusión de deportes no institucionalizados o programas de deportes de iniciación para activar la participación de los jóvenes y la propuesta de organizar los JOJ a un año "no olímpico" para garantizar un mayor posicionamiento mediático, comenzando por la cuarta edición de los JOJ de verano que en lugar de 2022 tengan cita en 2023 (COI, 2014c), no coincidiendo así con los Juegos Olímpicos de invierno de Pekín 2022. 
Con el objetivo de acercar el olimpismo a los jóvenes, el COI ha desarrollado una estrategia que parte de la experiencia en la organización de los JJOO y que se apoya en aquellos actores de la familia olímpica que han garantizado el éxito de ediciones pasadas. Así, la primera edición celebrada en Singapur en 2010, que tuvo un coste de 75,5 millones (Wong, 2011), involucró a 3.524 atletas, 205 Comités Olímpicos Nacionales, 9 socios patrocinadores y a 20.000 voluntarios alcanzando una audiencia televisiva de 247 millones de personas y 5,9 millones de visitas web, como podemos observar (Tabla 1). Por su parte, en los Juegos de Nankín 2014 se incrementó la participación de atletas, eventos y disciplinas deportivas, aunque se redujo el número de voluntarios. En lo referente a la actividad en medios y nuevas tecnologías, la audiencia televisiva en Nankín 2014 se triplicó respecto a Singapur 2010, y el tráfico web alcanzó los 39 millones de visitas. Por otro lado, en Innsbruck 2012, inherente a la naturaleza de las ediciones de invierno, tradicionalmente menos mediáticas y con menos recursos que las ediciones de verano (Angelini y Billings, 2010), participaron 1.022 atletas procedentes de 69 Comités Olímpicos Nacionales, 1.440 voluntarios ayudaron a la organización del evento y 900 medios acreditados fueron testigos de la primera edición de invierno los Juegos Olímpicos de la Juventud.

\section{Tabla 1.}

Datos generales durante los Juegos Olímpicos de la Juventud (2010-2014)

\begin{tabular}{|l|r|r|r|}
\hline & Singapur 2010 & Innsbruck 2012 & Nankín 2014 \\
\hline Deporte y organización & & & \\
\hline Disciplinas & 26 & 7 & 28 \\
\hline Eventos & 201 & 63 & 222 \\
\hline Atletas & 3.524 & 1.022 & 3.759 \\
\hline Comités Olímpicos Nacionales & 205 & 69 & 204 \\
\hline Voluntarios & 20.000 & 1.440 & 18.551 \\
\hline Medios y marketing & & & \\
\hline Medios acreditados & 1.768 & 900 & 1.832 \\
\hline Audiencia TV & 247.000 .000 & sin datos & 769.000 .000 \\
\hline Visitas Página Web & 5.900 .000 & 4.700 .000 & 39.000 .000 \\
\hline Patrocinadores TOP & 9 & 11 & 10 \\
\hline
\end{tabular}

Nota: Tabla de elaboración propia a partir de los informes oficiales del COI (2014d; 2012a; 2011; 2010b).

\subsection{El Movimiento Olímpico en las redes sociales}

Si los Juegos Olímpicos de Berlín en 1936 marcaron el inicio de la era de la televisión y su vinculación con el Movimiento Olímpico (Billings, 2008; Moragas et al., 1995), los Juegos de Atlanta 1996 pasaron a la historia por ser los primeros Juegos de la era internet que contaron con una página web propia que mostraba los resultados de las competiciones deportivas (Fernández 
Peña y Ramajo, 2014). Los posteriores Juegos de invierno de Nagano 1998 y Salt Lake City 2002 y las ediciones de verano de Sydney 2000 y Atenas 2004 fueron claves para un primer acercamiento de los actores que conforman la familia olímpica con la era de internet.

La revolución digital ha tenido un fuerte impacto en el Movimiento Olímpico. A principios de 2001 el 33,6\% de los Comités Olímpicos Nacionales ya disponían de página web (Moragas, 2001) y en Atenas 2004 la cadena norteamericana NBC empezó a ofrecer contenidos en vídeo en su versión digital (Fernández Peña y Ramajo, 2014). No obstante, aunque la incursión del Movimiento Olímpico en internet ha estado marcada por un crecimiento exponencial, también ha sufrido las consecuencias de la fuerte desigualdad que existe en el acceso a las tecnologías de la información desde sus inicios (Moragas, 2001). Mientras que en el año 2000 había 407 millones de personas con acceso a internet y un $6,71 \%$ de penetración global, a finales de 2015 la cifra ascendió a 3.300 millones de personas conectadas, un $46,4 \%$ de penetración mundial (Internet World Stats, 2015). Algunas de las alianzas contractuales por parte del COI con empresas como Samsung y Panasonic, que proporcionan productos y servicios de tecnología avanzada (Ferrand, Chappelet y Séguin, 2012), han afectado positivamente al desarrollo del Movimiento Olímpico en internet abriéndose a un público mucho más amplio y joven (Robart, 2008), mientras que la gestión de la venta de los derechos en internet y la protección de la marca olímpica en la red ha complejizado la consolidación de la "Olympic digital revolution” (Miah y García, 2012, p. 123).

El Comité Olímpico Internacional desembarca en la web 2.0 y en los medios sociales en los Juegos de Pekín en 2008 (Miah, García y Zhihui, 2008) y vende, por primera vez, los derechos audiovisuales para internet y televisión por separado (Hutchins y Mikosza, 2010). Este primer ensayo en las redes sociales se produce tras el acuerdo firmado por el COI y la plataforma YouTube para retransmitir vía online las competiciones deportivas en 78 territorios que no habían adquirido los derechos de los Juegos, lo cual advirtió sobre el futuro del consumo de los Juegos Olímpicos en la televisión (Miah y García, 2012). Para Hutchins y Mikosza (2010, p. 291):

"Beijing 2008 represents the mostly successful assertion of a market-based media logic over social production and exchange within digital networked communications environments, offering more sanctioned online Olympic content to international audiences than any previous Games".

["Pekín 2008 representa ante todo la exitosa reivindicación de un mercado de la lógica mediática por encima de la producción social e intercambio dentro de los ambientes de comunicaciones en red, ofreciendo más contenido Olímpico autorizado a las audiencias internacionales que cualquiera de los Juegos anteriores"].

Sin embargo, este primer acercamiento a las plataformas sociales experimentó problemas relacionados con "ambush marketing, broadcast media rights infringement, or unauthorized online communication by athletes or third parties" [ "ambush marketing, derechos televisivos o comunicaciones online no autorizadas de atletas o terceras partes"] (Traducido por los autores) (Ibídem).

Los Juegos Olímpicos de invierno de Vancouver 2010, celebrados durante el mes de febrero de 2010, suponen la primera experiencia del Comité Olímpico Internacional y del Comité Organizador en las plataformas Facebook y Twitter, inaugurando modelos que han seguido otras organizaciones deportivas, según el informe Social Networking and the Movement: social media analysis, opportunities and trends, elaborado por el CEO-UAB para el COI (Fernández Peña, 2011). Siguiendo este informe, tanto la página oficial de Vancouver 2010, gestionada por el Comité Organizador, como la página del Comité Olímpico Internacional superaron el millón de fans en Facebook alcanzando el mayor nivel de participación antes de la ceremonia de clausura. En el caso de Twitter, el 39,36\% de los contenidos generados por la cuenta del Comité Organizador Vancouver 2010 hizo alusión a resultados diarios de las competiciones, mientras que 
en el perfil oficial del COI predominó la difusión del ambiente vivido en los estadios y en sus alrededores (Fernández Peña, 2011, pp. 132-148).

Por su parte, los Juegos de Londres 2012 fueron catalogados oficialmente por los medios de comunicación como los primeros "Social Olympics" (Rooney, 2012, en Fernández Peña et al., 2014). Hasta Londres 2012 el COI había concebido las redes sociales como plataformas administradas de forma independiente que difunden el contenido ya publicado en la página web, sin fomentar la interacción con los públicos (Fernández Peña et al., 2011). Sin embargo, la variedad de canales (Google +, Tumblr, Instagram, Foursquare y Sina Weibo, entre otros), la alta participación del público y la dispersión de mensajes originados en Londres 2012 provocó un cambio de mentalidad en la concepción de las redes sociales por parte del COI. Este hecho propició la creación del Olympic Athletes' Hub, plataforma online diseñada para aglutinar la actividad generada por los atletas, que potencia y protege la marca digital de los Juegos Olímpicos (Lunden, 2012, en Fernández Peña et al., 2014). La actividad del COI en Facebook durante Londres 2012 se centró en aspectos simbólicos, como las ceremonias de apertura y clausura, en la difusión de los deportes olímpicos y en el papel de los atletas más populares, mientras que los contenidos publicados por el Comité Organizador tuvieron una perspectiva más local, enfocada en la promoción del evento y de la ciudad (Fernández Peña et al., 2014). La actividad en la página del COI en Facebook durante Londres 2012 estuvo marcada por la participación de 819.592 usuarios activos, de los cuales el 60,8\% fueron mujeres (Fernández Peña, Coromina y Pardo Gila, 2016).

No obstante, el Movimiento Olímpico ha experimentado un cambio de paradigma comunicativo con la llegada de las redes sociales que ha motivado la regularización de ciertas normas y recomendaciones para su uso. Esta normativa del COI se publicó en el documento IOC Social Media and Blogging Guidelines (COI, 2012b) con el objetivo de moderar la actividad generada por terceros durante los Juegos. Desde este documento se motiva a los atletas y al personal acreditado a publicar sus experiencias siempre que lo hagan en primera persona, nunca en calidad de periodista, ni revelando información confidencial o privada de cualquier otra persona u organización ni publicando aspectos relacionados con la competición que pudieran interferir en ella (Ibídem). Del mismo modo, el COI prohíbe la promoción y la difusión de marcas comerciales por parte de los atletas, así como aquellos contenidos de índole político o ideológico. Aunque estas medidas han sido criticadas por la restricción de libertad de expresión que pueden conllevar, el COI ha defendido que su único objetivo es garantizar la exaltación exclusiva del deporte y de la competición, protegiendo su marca y la de los actores que conforman la familia olímpica y siguiendo los principios fundamentales de la Carta Olímpica (COI, 2014c).

\section{3. Los Juegos Olímpicos de la Juventud y las redes sociales}

Las redes sociales están constituidas por un conjunto de actores (personas, organizaciones u otros grupos sociales) interconectados (Caldarelli y Catanzaro, 2014; Canals, 2012; Wasserman y Faust, 2013) dentro de una estructura de relaciones originada por vínculos de amistad, de colaboración o de otro tipo de interés (Fresco García, Marqués Sánchez y Paunero, 2014). Son ecosistemas complejos (Christakis y Folder, 2010) que requieren del análisis de su estructura para ser comprendidos y gestionados al presentar comportamientos propios de sistemas emergentes (Johnson, 2003). Estos sistemas siguen el principio de homofilia, es decir, la tendencia a relacionarse entre sí que despliegan los actores de una red que comparten género, idioma, origen étnico, clase social o valores (Kadushin, 2013). Los medios sociales basados en internet son medios interactivos que forman parte de un todo, en los cuales cada usuario es un emisor comunicativo con capacidad para crear y distribuir contenidos (Fernández Peña et al., 2014). Estos contenidos generados por los usuarios en estas plataformas son más eficaces desde el punto de vista comunicativo entre los jóvenes que los contenidos producidos por los medios 
tradicionales (Fernández Peña, 2009). En este entorno es clave el término engagement, que hace referencia a "la participación de los públicos, a su grado de implicación en relación con las publicaciones de terceros, una organización, empresa o individuo" (Fernández Peña et al., 2014, p. 155), revelándose como uno de los principales objetivos de cualquier actor presente en los medios sociales.

El objetivo del COI es involucrar al público en general y a los jóvenes en particular aprovechando "la gran capacidad estratégica de los medios sociales y nuevos dispositivos para conectar con los usuarios difundiendo los valores e ideales olímpicos" (Fernández Peña et al., 2014, p. 153). El nacimiento de los Juegos de la Juventud coincide con la emergencia de las redes sociales. Especialmente con el crecimiento y consolidación de Facebook, la plataforma social más popular que contaba con 482 millones de usuarios activos al mes en 2010 (Facebook, 2012) y alcanzó los 1.550 millones de usuarios únicos al mes en septiembre de 2015 (Facebook, 2015). Según Fernández Peña (2011, p. 218), "Los Juegos Olímpicos de la Juventud son un evento cuya estrategia de comunicación y filosofía se centra en los jóvenes, que son más activos en las redes sociales".

Como podemos observar (Tabla 2), existe una evolución en el uso de los medios sociales para las tres ediciones analizadas de los Juegos Olímpicos de la Juventud. Mientras que en Singapur 2010 se publicaron contenidos en cuatro redes sociales (Facebook, Flickr, Twitter y YouTube) y en Innsbruck 2012 se añadió a éstas la red social Sina Weibo, en Nankín 2014 los JOJ han tenido presencia en diez plataformas online (Facebook, Flickr, Google+, Instagram, Sina Weibo, Tencent WeChat, Tencent Weibo, Tumblr, Twitter y YouTube).

Destaca la evolución en el uso de Facebook, que pasó de 82.745 fans totales en Singapur 2010 a 420.000 en Nankín 2014 (COI, 2010b; COI 2014d). Durante Singapur 2010, 140 posts publicados involucraron a 3.677 usuarios activos, en Innsbruck 2012, 83 posts motivaron la participación de 1.321 usuarios, mientras que en Nankín 2012, 112 posts activaron a 8.666 usuarios únicos (Pardo Gila, 2015). Los contenidos publicados en Facebook que generaron mayor nivel de engagement entre los usuarios fueron fotografías de las ceremonias de apertura y clausura (Ibídem). En el caso de Twitter, Nankín 2014 alcanzó los 24.000 followers (seguidores), por los 5.982 con los que contó Singapur 2010, edición en la que YouTube tuvo un papel principal obteniendo casi un millón y medio de visitas en los 442 vídeos publicados en su cuenta oficial (COI, 2010b; COI 2014d). Sin embargo, la relevancia de Flickr durante Singapur 2010, donde se publicaron más de 14.000 fotografías que tuvieron casi tres millones de visitas, y durante Innsbruck 2012, con más de 7.800 fotografías registradas (COI, 2010b; COI, 2012a), se aminoró en la edición de Nankín 2012. Este hecho pudo estar provocado por el auge de otra plataforma dedicada a la publicación de fotografías, Instagram, donde las 578 publicaciones del perfil oficial de Nankín 2014 fueron seguidas de 30.000 seguidores (COI 2014d).

Aunque las redes sociales comportan una concepción totalizadora de un mundo interconectado a nivel global, éstas sobreviven al coexistir en un ambiente local (Martel, 2014). Es el caso de Nankín 2014, donde el crecimiento del uso en las redes sociales está también relacionado con la explotación local de los canales digitales en China a través de plataformas como Sina Weibo - con presencia también durante Innsbruck 2012-, Tencent WeChat y Tencent Weibo -en ésta última se publicaron 5.962 post y se alcanzaron 600.000 seguidores-. Sin embargo, el mayor éxito de los Juegos Olímpicos de la Juventud en las redes sociales llegó a través de la campaña digital \#YOGSelfie lanzada a la vez en diferentes plataformas como Twitter, Facebook e Instagram, con un alcance total de 500 millones de usuarios y 58 millones de publicaciones en la red social china Sino Weibo (COI, 2014b). 
Tabla 2.

Presencia en los perfiles oficiales de los Comités Organizadores Juegos Olímpicos de la Juventud en las redes sociales (2010-2014):

\begin{tabular}{|l|c|c|c|}
\hline & Singapur 2010 & Innsbruck 2012 & Nankín 2014 \\
\hline Facebook & $\checkmark$ & $\checkmark$ & $\checkmark$ \\
\hline Flickr & $\checkmark$ & $\times$ & $\checkmark$ \\
\hline Google+ & $\times$ & $\times$ & $\checkmark$ \\
\hline Instagram & $\times$ & $\checkmark$ & $\checkmark$ \\
\hline Sina Weibo & $\times$ & $\times$ & $\checkmark$ \\
\hline $\begin{array}{l}\text { Tencent } \\
\text { WeChat }\end{array}$ & $\times$ & $\times$ & $\checkmark$ \\
\hline $\begin{array}{l}\text { Tencent } \\
\text { Weibo }\end{array}$ & $\times$ & $\checkmark$ & $\checkmark$ \\
\hline Tumblr & $\checkmark$ & $\checkmark$ & $\checkmark$ \\
\hline Twitter & $\checkmark$ & $\times$ & $\checkmark$ \\
\hline YouTube & $\times$ & $\times$ & $\checkmark$ \\
\hline
\end{tabular}

Nota: Elaboración propia a partir de los informes oficiales del COI (2010b; 2012a; 2014d).

\section{Discusión y conclusiones}

El desembarco del Comité Olímpico Internacional en internet a mediados de los años noventa y la posterior llegada de las redes sociales en los primeros años del nuevo milenio, ha provocado una serie de importantes cambios que han alterado las formas de comunicación entre los actores que confluyen en el Movimiento Olímpico. Los cambios producidos con motivo de la comercialización de los derechos de televisión a raíz de la aparición de plataformas como YouTube, la gestión de grandes comunidades de fans surgidas en Facebook e Instagram a partir de la creación de contenidos multimedia, el acercamiento entre deportistas y fans que ha proporcionado la inmediatez de la plataforma Twitter o la llegada al mercado asiático a través de redes sociales como Sina Weibo y Tencent WeChat han permitido la expansión del olimpismo en el mundo.

El objetivo del Movimiento Olímpico en las redes sociales debe consistir en involucrar a los jóvenes haciéndolos partícipes en la construcción de un nuevo mensaje olímpico, enfocado en los valores tradicionales del olimpismo, pero adaptado al lenguaje de los nuevos medios de internet. La capacidad y la naturaleza de los medios sociales y los nuevos dispositivos, así como los cambios de hábito en el consumo audiovisual, ofrecen un abanico de posibilidades para el diseño y desarrollo de contenidos y herramientas que pueden permitir una participación activa a través de la comunicación más directa con los actores protagonistas. Además, como precursor de avances tecnológicos el Movimiento Olímpico debe entender la era de los nuevos medios como 
una oportunidad histórica para aprovechar su posición privilegiada adelantándose a los cambios y beneficiándose de sus propias innovaciones para consolidar su poder de transmisión para ser pionero, una vez más, en el campo de la comunicación.

Si concebimos internet no como un fenómeno principalmente global, sino como algo enraizado inherentemente a un territorio local (Martel, 2014), el COI debería reflexionar sobre aquellos elementos básicos que tienen que ver con el perfil específico del receptor al que se dirigen sus contenidos: ¿qué idioma habla? ¿Qué edad tiene? ¿A qué clase social pertenece? Según Martel (2014, p. 22), "internet no suprime los límites geográficos tradicionales, ni disuelve las identidades culturales, ni allana las diferencias lingüísticas, sino que las consagra". Esta idea puede aportar algo de luz a las instituciones deportivas globales como el Comité Olímpico Internacional a la hora de diseñar estrategias en las redes sociales. La diseminación y segmentación de los mensajes por idioma, edad, grupo social o género y las oportunidades que brinda el big data para conocer el comportamiento de los usuarios (Mayer-Schönberger y Cukier, 2013) puede ser un método eficaz para conseguir una comunicación más directa e individualizada y una participación más activa con los usuarios, además de un canal óptimo para monetizar la marca digital olímpica.

Sin embargo, todos estos retos y oportunidades han de ser coherentes con la comercialización de los Juegos Olímpicos que garantizan su viabilidad económica. En esta línea, se deben respetar los principios fundamentales recogidos en la Carta Olímpica para proteger la marca olímpica y la de todos los actores que confluyen en el sistema olímpico: deportistas de élite, Comités Organizadores, Comités Olímpicos Nacionales, federaciones, ligas profesionales, patrocinadores, televisiones, medios acreditados, voluntarios y espectadores.

Como adalid del Movimiento Olímpico, el Comité Olímpico Internacional goza de una oportunidad histórica para conectar a la generación nativa en internet con la filosofía del olimpismo concebida desde sus orígenes por Pierre de Coubertin como herramienta pedagógica universal para construir un mundo mejor educando a la juventud en valores como el esfuerzo, la tolerancia y el respeto.

\section{Referencias Bibliográficas}

- Angelini, J. R. y Billings, A. C. (2010). An Agenda That Sets the Frames: Gender, Language, and NBC's Americanized Olympic Telecast. Journal of Language and Social Psychology, 29, 363-385. http://dx.doi.org/10.1177/0261927X10368831

- Billings, A. C. (2008). Olympic media: inside the biggest show on television. New York: Routledge.

- Billings, A. C. (2010). La comunicación en el deporte. Barcelona: Editorial UOC.

- Caldarelli, G. y Catanzaro, M. (2014). Redes: una breve introducción. Madrid: Alianza editorial.

- Canals, A. (2012). Xarxes socials: fonaments i aplicacions. Barcelona: Editorial UOC.

- Castells, M. (2001). La Era de la información: economía, sociedad y cultura. Madrid: Alianza. 
- Christakis, N. A. y Fowler, J. H. (2010). Conectados: el sorprendente poder de las redes sociales y cómo nos afectan. Madrid: Taurus/Santillana.

- Coubertin, P. (2012). La educación inglesa. Citius, Altius, Fortius: Humanismo, Sociedad y Deporte: Investigaciones y ensayos, 5(1). Centro de Estudios Olímpicos de la UAM. Madrid. 133-151.

- Durántez Corral, C. (s.f.). Academias Olímpicas Nacionales. Madrid: Academia Olímpica Española.

- Ferrand, A., Chappelet, J. L. y Séguin, B. (Coords.) (2012). Olympic Marketing. London: Routledge.

- Fernández Peña, E. (2009). The Youth Olympics and the media: towards a new relation. En K. Georgiadis (Ed), Proceedings of the International Olympic Academy $8^{\text {th }}$ International Session for Educators and Officials of Higher Institutes of Physical Education (pp. 200-202). Olimpia, Grecia: Academia Olímpica Internacional.

- Fernández Peña, E. (et al.) (eds.) (2011). An Olympic Mosaic: Multidisciplinary Research and Dissemination of Olympic Studies. CEO-UAB: 20 Years. Barcelona: CEO-UAB y Ajuntament de Barcelona.

- Fernández Peña, E., Coromina, Ò. y Pardo Gila, J. M. (2016). The nature of engagement on Facebook during the London 2012 Olympic Games: an insight into the public's participation in terms of language and gender. [En imprenta].

- Fernández Peña, E. y Ramajo, N. (2014). La comunicación en el deporte global: los medios y los Juegos Olímpicos de verano (1894-2012). Historia Y Comunicación Social, 19, $703-$ 714. http://dx.doi.org/10.5209/rev hics.2014.v19.45171

- Fernández Peña, E., Ramajo, N. y Arauz, M. (2014). Social Media in the Olympic Games: Actors, Management and Participation. Sport and New Media. En: Billings, A. y Hardin, M. (Eds.), Handbook of Sport and New Media. New York: Routledge. pp. 153-164.

- Fresno García, M. del, Marqués Sánchez, P. y Paunero, D. S. (2014). Conectados por redes sociales: introducción al análisis de redes sociales y casos prácticos. Barcelona: Editorial UOC.

- Hutchins, B. y Mikosza, J. (2010). The Web 2.0 Olympics: Athlete blogging, social networking and policy contradictions at the 2008 Beijing Games. Convergence: The International Journal of Research into New Media Technologies, 16(3), 279-297 http://dx.doi.org/10.1177/1354856510367618

- Jackson, R. (2014). "El nacimiento de los Juegos Olímpicos de la Juventud". En: Manual de administración deportiva, Canadá: Solidaridad Olímpica. Comité Olímpico Internacional. p. 55-58.

- Jenkins, H. (2008). Convergence culture: la cultura de la convergencia de los medios de comunicación. Barcelona: Paidós. 
- Johnson, S. (2003). Sistemas emergentes: o qué tienen en común hormigas, neuronas, ciudades y software. México, D.F.: Fondo de Cultura Económica.

- Judge, L. W., Lee, D., Surber, K., Bellard, D., Petersen, J., Ivan, E., y Kim, H. J. (2013). The Promotion and Perception of the Youth Olympic Games: A Korean Perspective. The ICHPER-SD Journal of Research in Health, Physical Education, Recreation, Sport \& Dance, $8(2), 12-18$.

- Judge, L. W., Petersen, J. y Lydum, M. (2009). The Best Kept Secret in Sports: The 2010 Youth Olympic Games. International Review for the Sociology of Sport, 44, 173-191 http://dx.doi.org/10.1177/1012690209335939

- Kadushin, C. (2013). Comprender las redes sociales: teorías, conceptos y hallazgos. Madrid: Centro de Investigaciones Sociológicas.

- Levine, F., Locke, C., Searls, D. y Weinberger, D. (2008). El Manifiesto Cluetrain. Barcelona: Deusto.

- Makris, A. y Georgiadis, K. (2013). Los Juegos Olímpicos de la Juventud: una nueva institución del Movimiento Olímpico para la difusión de los valores educativos del olimpismo. Citius, Altius, Fortius. Humanismo, sociedad y deporte: Investigaciones y ensayos 6 (2), Centro de Estudios Olímpicos de la Universidad Autónoma de Madrid. Pgs. 76-95.

- Martel, F. (2014). Smart: internet(s): la investigación. Madrid: Taurus.

- Mayer-Schönberger, V. y Cukier, K. (2013). Big data: la revolución de los datos masivos. Madrid: Turner.

- Miah, A. y García, B. (2012). The Olympics: The Basics. Nueva York: Routledge.

- Miah, A., García, B. y Zhihui, T. (2008). We are the Media: Non-Accredited Media and Citizen Journalists at the Olympic Games, En: Price, M. E. y Dayan, D. (eds). Owning the Olympics: Narratives of the New China. Ann Arbor. The University of Michigan Press. pp. 320-345.

- Moragas, M. (2001). Internet and the Olympic Movement. Trabajo presentado en el 11th International Association for Sport Information (IASI) World Congress, IOC, Lausane. Recuperado de www.olympicstudies.uab.es/pdf/OD012_eng.pdf

- Moragas, M., Rivenburgh, N. K. y Larson, J. F. (1995). Television in the Olympics. Londres: John Libbey.

- Pardo Gila, J. M. (2015). The engagement on Facebook during the Youth Olympic Games of Singapore 2010, Innsbruck 2012 and Nanjing 2014: comparative analysis and participation of users from a language and gender perspective. Trabajo presentado en el XXII International Seminar on Olympic Studies for Postgraduate Students, Academia Olímpica Internacional, Olimpia, Grecia.

- Ruiz Olabuénaga, J. (2012). Metodología de la Investigación Cualitativa. Deusto: Universidad de Deusto. 
- Wasserman, S. y Faust, K. (2013). Análisis de redes sociales: métodos y aplicaciones. Madrid: Centro de Investigaciones Sociológicas.

- Wong, D. (2011). The Youth Olympic Games: Past, Present and Future. The International Journal of the History of Sport, 28 (13), 1831-1851. http://dx.doi.org/10.1080/09523367.2011.594687

\subsection{Artículos en publicaciones web}

- Comité Olímpico Internacional (2007). 1st Summer Youth Olympic Games in 2010. Lausana: Comité Olímpico Internacional. Disponible en http://goo.gl/dcFDP8 [Consulta: 2015, 3 de diciembre]

- Comité Olímpico Internacional (2009). What is YOG? Disponible en http://goo.gl/tFbuqY [Consulta: 2015, 10 de diciembre]

- Comité Olímpico Internacional (2010a). Culture and Education Programme (CEP) Explanatory Brochure. Lausana: Comité Olímpico Internacional. Disponible en https://goo.gl/gMcIqL [Consulta: 2015, 10 de diciembre]

- Comité Olímpico Internacional (2010b). Official Report Singapore 2010 Youth Olympic Games. Lausana: Comité Olímpico Internacional. Disponible en http://goo.gl/EfKYXT [Consulta: 2015, 3 de diciembre]

- Comité Olímpico Internacional (2011). The Youth Olympic Games. Lausana: Comité Olímpico Internacional. Disponible en http://goo.gl/396K6A [Consulta: 2015, 3 de diciembre]

- Comité Olímpico Internacional (2012a). Official Report Innsbruck 2012 Youth Olympic Games. Lausana: Comité Olímpico Internacional. Disponible en http://goo.gl/FG7cgS [Consulta: 2015, 12 de diciembre]

- Comité Olímpico Internacional (2012b). IOC Social Media, Blogging and Internet Guidelines for Participants and Other Accredited Persons at the London 2012 Olympic Games. Lausana: Comité Olímpico Internacional. Disponible en http://goo.gl/CYmRch [Consulta: 2015, 12 de diciembre]

- Comité Olímpico Internacional (2013). 2nd IOC Young Reporters Programme, Nanjing 2014 Nanjing Youth Olympic Games, 16-28 August 2014. Disponible en http://goo.gl/uKUce8 [Consulta: 2015, 3 de diciembre]

- Comité Olímpico Internacional (2014a). The Youth Olympic Games: vision and principles. Lausana: Comité Olímpico Internacional. Disponible en http://goo.gl/FXZjoU [Consulta: 2016, 21 de enero]

- Comité Olímpico Internacional (2014b). IOC Annual Report 2014. Lausana: Comité Olímpico Internacional. Disponible en http://goo.gl/8wXLCG [Consulta: 2016, 21 de enero]

- Comité Olímpico Internacional (2014c). Olympic Agenda 2020: 20+20 recommendations. Lausana: Comité Olímpico Internacional. Disponible en http://goo.gl/UWSV2j [Consulta: 2016, 21 de enero] 
- Comité Olímpico Internacional (2014d). Official Report Nanjing 2014 Youth Olympic Games. Lausana: Comité Olímpico Internacional. Disponible en http://goo.gl/2njqQ8 [Consulta: 2015, 12 de diciembre]

- Comité Olímpico Internacional (2014e). The Youth Olympic Games, learn \& share beyond the field of play. Disponible en http://goo.gl/aVgGJY [Consulta: 2015, 12 de diciembre]

- Facebook (2012). "Facebook 2012 Report Annual". En: Facebook.com. Disponible en https://goo.gl/4QKqpD [Consulta: 2015, 14 de noviembre]

- Facebook (2015). "Facebook Reports First Quarter 2015 Results”. En: Facebook.com Disponible en http://goo.gl/XmjW7L [Consulta: 2016, 23 de enero]

- Fernández Peña, E. (ed.) (2011). Social Networking and the Olympic Movement: Social media analysis, opportunities and trends. Report commissioned by the International Olympic Committee. Barcelona: Centro de Estudios Olímpicos de la Universidad Autónoma de Barcelona (CEO-UAB). Disponible en https://goo.gl/4WS76a [Consulta: 2015, 25 de noviembre]

- Internet World Stats (2015). World Internet Users and 2015 Population Stats. Disponible en http://goo.gl/VKYBkM [Consulta: 2016, 16 de enero]

- Lenhart, A, (2015). “Teen, Social Media and Technology Overview 2015”. En: Pew Research Center. Disponible en http://goo.gl/23C6WP [Consulta: 2016, 2 de febrero]

- Makris, A y Georgiadis, K. (2013) Los Juegos Olímpicos de la Juventud: una nueva institución del movimiento olímpico para la difusión de los valores educativos del olimpismo. Citius, Altius, Fortius, Humanismo, Sociedad y Deporte: Investigaciones y ensayos, 6(2). Centro de Estudios Olímpicos de la UAM. Madrid. Pp.76-95.

- Moragas, M., Kennett, C., y García Sedó, R. (2004). El Impacto de Internet en los medios de comunicación y la industria del deporte. Bellaterra: Centro de Estudios Olímpicos de la Universidad Autónoma de Barcelona (CEO-UAB). Disponible en http://goo.gl/jsRGXZ [Consulta: 2015, 14 de noviembre]

- Robart, M. (2008). "Olympics Depending on Technology to Attract Younger Viewers". En: TMC Net, 07 de agost 